\title{
A ROBUST CORNER MATCHING TECHNIQUE
}

\author{
Mohammad Awrangjeb and Guojun Lu \\ Gippsland School of Information Technology, Monash University, Churchill Vic 3842, Australia \\ \{Mohammad.Awrangjeb, Guojun.Lu\}@infotech.monash.edu.au
}

\begin{abstract}
In contour-based corner detectors, absolute curvature values of many corners either remain unaltered or change slightly under affine transformations. Moreover, affine-length of a curve is relatively invariant to affine transformations. This paper presents a novel corner matching technique using corners detected by contour-based detectors. For each corner we use its position, absolute curvature value, and affine-lengths between this corner and other corners on the same curve. The iterative matching procedure tries to find three corner matches with minimum absolute curvature difference to calculate affine transformation parameters. Original corners are transformed with the estimated parameters prior to matching with the test corner set.
\end{abstract}

\section{INTRODUCTION}

The problem of how to automatically estimate correspondences of feature points between two images while avoiding mismatches is a fundamental problem in computer vision and has been extensively studied for a couple of decades [1]. Although a significant number of methods have been developed, none is general and reliable enough to cope with all possible cases due to difficulties in feature selection and correspondence matching [2]. The solution is computationally intensive and difficult due to following reasons: first, additional points may be detected in the test image due to noise or clutter; second, desirable points may be missed in the test image due to noise or occlusion; and finally, the corner strength associated with some numerical value may be uncertain [3].

Feature matching techniques can be broadly categorized into two. In the first category, each feature is associated with its local neighborhood and the matching procedure involves neighborhood matching [4]. These techniques are robust but computationally expensive and require large storage for descriptors. In the second category, each feature (corner) is associated with information like its curvature but do not use neighbor intensity values for matching $[1,2,5-9]$. This paper presents a corner matching technique of the second category.

In the second category, the matching problem was first formulated as the sub-graph isomorphism problem to find the maximal clique [5], which is an NP-complete problem [3]. The problem was later formulated as an optimization problem and either relaxation technique [6] or binary Hopfield network [7] was applied for cost minimization. A similar approach based on the fuzzy inference procedure was proposed in [8]. A solution using the Hausdorff distance which determined the degree of mismatch between two sets of feature points is in [9]. However, the Hausdorff distance is too sensitive to noises or outliers and is not invariant to geometric transformations. The method in [1] used a similarity measure based on the corner strength. It first matched local feature groups and then estimated the affine transformation by global matching. Zhou et al. [2] proposed a novel corner matching technique based on the Delaunay triangulations which were formed among corners (DT-based matching). The corner correspondence was established based on the observation that the interior angles of the Delaunay triangles completely and uniquely characterized the corners and their values were not affected much by rotation, uniform scale change, and translation. At the matching stage, the most similar triangle pairs were obtained and then their edges were extended circularly until all matching corners were triangulated and mismatching corners were discarded. The DTbased matching was very much promising; however, it would fail under aspect-ratio change and general affine transformations where interior angles of Delaunay triangles are affected.

Most of the above solutions [1, 5-9] were computationally expensive and their performance was questionable even in small scale change. This paper presents a novel corner matching technique which is robust to affine transformations as well as to noising and lossy compression. The proposed method can be used with contour-based corner detectors depending on the availability of the required information. In particular, here we use it with our previously proposed affine resilient curvature scale-space corner detector (ARCSS detector) [10]. During corner detection this corner detector extracts planar curves from images. For corner matching we associate each corner with information like its curvature and affine-lengths between this corner and other corners on the same curve (AL-based matching). An iterative procedure tries to estimate affine transformation parameters between original and test images. Once estimated transformation parameters are found, all original corners are transformed prior to matching with the test corner set.

\section{THE ARCSS CORNER DETECTOR}

The ARCSS corner detector [10] extracts edges in the gray-scale image using the Canny edge detector [11]. It then parameterizes each curve (edge) with the affine-length. In order to eliminate noise, it convolves each parameterized curve using the Gaussian kernel in one of three medium scales based on the curve's affinelength. Thereafter, it calculates absolute curvature value on each point of the smooth curves and considers curvature maxima points as corners. Both round and false corners are removed using thresholds. Finally, corners are tracked down to the finest scale in order to improve localization.

For a given parametric vector equation of a planar curve $\Gamma(t)=(x(t), y(t))$, the curvature is defined as [10]:

$$
\kappa(t)=\frac{\dot{x}(t) \ddot{y}(t)-\ddot{x}(t) \dot{y}(t)}{\left(\dot{x}^{2}(t)+\dot{y}^{2}(t)\right)^{3 / 2}},
$$


where $\dot{x}(t)$ and $\dot{y}(t)$ are first and $\ddot{x}(t)$ and $\ddot{y}(t)$ are second order derivatives. The affine-length $\tau$ between two points $P_{1}$ and $P_{2}$ is

$$
\tau=\int_{P_{1}}^{P_{2}}(\dot{x}(t) \ddot{y}(t)-\ddot{x}(t) \dot{y}(t))^{1 / 3} d t,
$$

which is absolutely invariant to rotation, but relatively invariant to affine transformations (see Section 3.1). When the curve is parameterized using $\tau$ it can be shown that [10]

$$
\dot{x}(\tau) \ddot{y}(\tau)-\ddot{x}(\tau) \dot{y}(\tau)=1 \text {. }
$$

Therefore, the curvature on the affine-length parameterized curve in the scale-space is given by according to (1) as

$$
\kappa(\tau, \sigma)=\frac{1}{\left(\dot{\mathrm{X}}^{2}(\tau, \sigma)+\dot{\mathrm{Y}}^{2}(\tau, \sigma)\right)^{3 / 2}},
$$

where according to the property of the Gaussian convolution

$$
\dot{\mathrm{X}}(\tau, \sigma)=x(\tau) * \dot{g}(\tau, \sigma) \text { and } \dot{\mathrm{Y}}(\tau, \sigma)=y(\tau) * \dot{g}(\tau, \sigma),
$$

where $g(., \sigma)$ denotes the Gaussian kernel of width (scale) $\sigma$ and * denotes the convolution.

Since the curvature defined in (4) is not invariant to affine transformations, the absolute curvatures of corners may change due to affine transformations; however, for many corners they either remain unaffected or change slightly. Fig. 1 shows that the ARCSS detector has on the average more than 12 repeated corners within curvature difference 0.2 ; however, we need only 3 of them to estimate affine transformations parameters (see Section 3.1).

\section{PROPOSED CORNER MATCHING}

The feature correspondence is straightforward if no geometrical transformation is occurred. Any geometrical transformation causes shifting of original pixel locations. As a result, the feature correspondence may be unsuccessful. Therefore, the aim of the proposed corner matching technique is to estimate the affine transformation parameters prior to corner correspondence. To do that, for each corner we use its position, absolute curvature, and affine-lengths between this corner and other corners on the same curve. The iterative matching procedure finds at least three matching corners with the minimum curvature difference. If the matching corners are non-collinear on each image, we find the affine transformation matrix between matching corners. Then we transform all corners in the original image using the estimated transformation matrix and match with the test corner set. For a successful corner correspondence, according to the average repeatability of the ARCSS detector [10], we assume that more than $30 \%$ corners should be truly matched.

\subsection{Affine Transformations and Invariance of Affine-length}

The general affine transformation of a point $P=(x, y)$ is

$$
P_{a}=A \cdot P+T,
$$

where $P_{a}=\left(x_{a}, y_{a}\right)$ is the transformed point, $T=\left[\begin{array}{ll}t_{x} & t_{y}\end{array}\right]^{t}$ is the translation matrix, and $A$ is the affine transformation matrix. When $A$ is comprised of rotation and scale then it can be defined as

$$
A=\left[\begin{array}{ll}
a & c \\
b & d
\end{array}\right]=\left[\begin{array}{rr}
s_{x} \cos \theta & -s_{x} \sin \theta \\
s_{y} \sin \theta & s_{y} \cos \theta
\end{array}\right],
$$

where $\theta$ is rotation angle and $s_{x}$ and $s_{y}$ are scale factors along $x$ and $y$-axis. Therefore, there are six unknowns in (6) and we need at



Fig. 1. Average number of repeated corners by the ARCSS detector in affine transformations (using images in Section 4).

least three matching corner pairs to find the transformation parameters. Once we know the six unknowns, we can find translation parameters from two unknowns and other parameters as

$$
\theta=\tan ^{-1}(b / d), s_{x}=\sqrt{a^{2}+c^{2}} \text {, and } s_{y}=\sqrt{b^{2}+d^{2}} .
$$

By using (2) \& (6), the affine-length $\tau_{a}$ of a transformed curve is

$$
\tau_{a}=\tau \operatorname{det}(A)^{1 / 3}=\tau\left(s_{x} s_{y}\right)^{1 / 3},
$$

where $\tau$ is the affine-length of the original curve. This relation shows that the affine-length is absolutely invariant to rotation, but relatively invariant to scale, hence to affine transformations.

\subsection{Affine-length-based Matching Procedure}

The inputs to the proposed AL-based iterative corner matching procedure are two corner sets $C_{o}$ and $C_{t}$ of original and test images respectively. Each corner is associated with its absolute curvature value, corresponding curve number, and the affine-length of its predecessor on the same curve. The output of the procedure is the estimated transformation matrix with maximum corner matches.

The AL-based iterative corner matching procedure consists of the following steps to estimate affine transformation parameters: 1. Initialize maximum curvature difference $d=0$, minimum corner matches $m=3$, and output $O=[]$.

2. Find the set of candidate matching corner pairs $C_{c}$ between $C_{o}$ and $C_{t}$ with curvature difference less than or equal to $d$.

3. For each candidate pair $\left(c_{o}, c_{t}\right)$ in $C_{c}$, add the new corner pair $\left(c_{o n}, c_{t n}\right)$ to $C_{c}$ if the affine-length ratio of corresponding curve segments (i.e., $c_{o} \rightarrow c_{o n} \& c_{t} \rightarrow c_{t n}$ ) is within the range $[l h]$.

4. For (next) three non-collinear corner pairs in $C_{c}$, find $A$ and $T$.

5. Transform all corners in $C_{o}$ using $A$ and $T$; i.e., $C_{o t}=A . C_{o}+T$.

6. Find the set of matching corner pairs $C_{m}$ between $C_{o t}$ and $C_{t}$.

7. If $\left(\left|C_{m}\right|>m\right)$, then $m=\left|C_{m}\right|, O=\left[C_{m}, A, T\right]$.

8. If $\left(\left|C_{m}\right|>0.3 \times\left|C_{o}\right|\right)$ return $O$, else Go to step 4 .

9. $d=d+0.02$. If $(d>T h)$, then return $O$, else go to step 2 .

In step 1 , minimum corner matches $m$ is initialized to 3 , because for any three non-collinear corner pairs there are already 3 matches. A preprocessing is carried out on $C_{o}$ and $C_{t}$ separately so that maximum 3 corners will have the same curve number in each input corner set. The preprocessing significantly reduces the number of false candidate pair additions in step 3 for each false candidate match in step 2. In step 3, corners $c_{o}$ and $c_{o n}$ should have the same curve number in $C_{o}$ and corners $c_{t}$ and $c_{t n}$ should have the same curve number in $C_{t}$. The procedure is further speeded up by using a look up (hash) table for combinations of corner pairs visited in step 4. In order to allow scale factors (both uniform and non-uniform) in the range [ 0.52 .0$]$ the range of affine-length ratio 


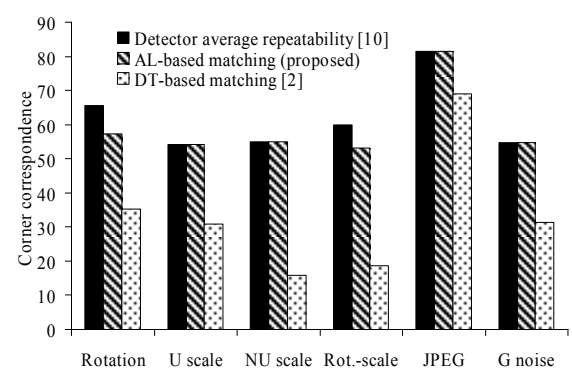

Fig. 2. Overall corner correspondence by matching methods.

(a)
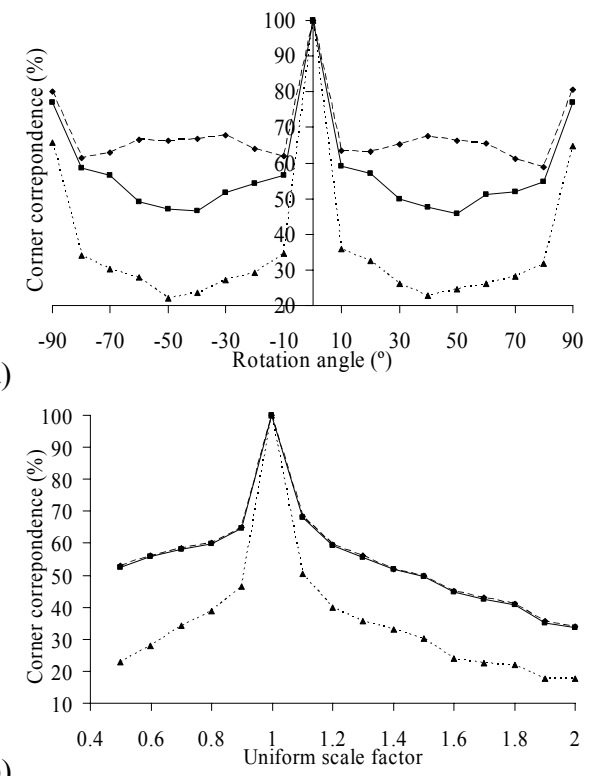

(b)

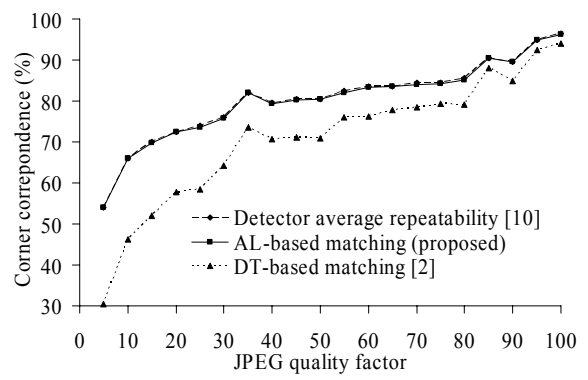

(c)

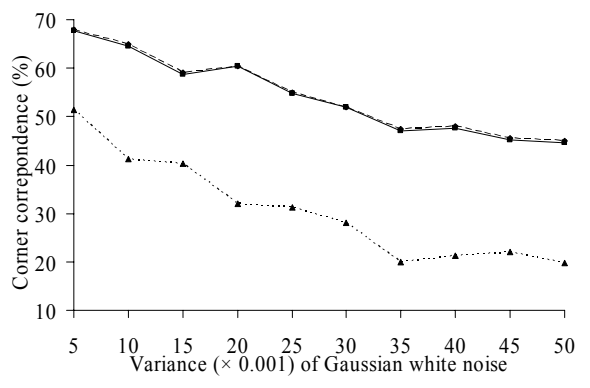

(d)

Fig. 3. Corner correspondence under different attacks.

is set as $[l h]=\left[\begin{array}{ll}0.63 & 1.59\end{array}\right]$ according to (9). While finding corner matches in step 6, we allow a maximum mean-square-error of 9 (3 pixels). Moreover, we set a threshold for $d$ as $T h=0.2$ (see Fig. 1).
Note that two loops (steps $4-8$ and 2-9) to ensure the best match may make the procedure exhaustive. However, we set a loop breaking condition in step 8 to avoid exhaustive search. Because, more than $30 \%$ corner matches (lower bound) is a reasonable condition based on the repeatability of the ARCSS detector [10]. Moreover, two different images if they are not originated from the same original should not have more than $30 \%$ matches, since corners reside randomly in images. In experiments, we observed the same and in most of the cases the best matches were found in first few iterations of the outer loop (steps $2-9$ ).

\section{PERFORMANCE STUDY}

We used MATLAB 7.0 to implement the proposed AL-based and existing DT-based [2] matching procedures with the ARCSS detector. We had total 23 different real original $512 \times 512$ grayscale images [12] and their total 7590 transformed images of six categories as test images. We rotated each original image at 18 different angles $\theta$ in $\left[-90^{\circ}+90^{\circ}\right]$ at $10^{\circ}$ apart, excluding $0^{\circ}$. We resized images using uniform (U) scale factors $s_{x}=s_{y}$ in [ $\left[\begin{array}{ll}0.5 & 2.0\end{array}\right]$ at 0.1 apart, excluding 1.0. We also resized using non-uniform (NU) scale factors $s_{x}$ in $\left[\begin{array}{lll}0.7 & 1.5]\end{array}\right.$ and $s_{y}$ in [ 0.51 .8$]$ at 0.1 apart, excluding the cases when $s_{x}=s_{y}$. We further used combined transformations (rot.-scale): $\theta$ in $\left[-30^{\circ}+30^{\circ}\right]$ at $10^{\circ}$ apart, excluding $0^{\circ}$, followed by uniform or non-uniform scale factors $s_{x}$ and $s_{y}$ in $[0.81 .2]$ at 0.1 apart. We compressed images using JPEG compression at 20 quality factors in [5 100] at 5 apart. We also added zero mean white Gaussian $(\mathrm{G})$ noise at 10 variances in [0.005 0.05 ] at 0.005 apart. Therefore, we had total 414 rotated, 345 uniform scaled, 2691 non-uniform scaled, 3450 rotated and scaled transformed images. We also had 460 JPEG compressed and 230 Gaussian noised images. Note that transformations comprising rotations were also followed by cropping such that the outer black parts were disappeared. Consequently, many detected corners in original images were cropped off in test images for the transformations involving rotations.

For a fair and error free comparisons we used the following metric to measure the corner correspondence:

$$
C C=\frac{N_{m}}{2}\left(\frac{1}{N_{o}}+\frac{1}{N_{t}}\right),
$$

where $N_{o}$ and $N_{t}$ are the number of corners detected in original and test images and $N_{m}$ is the number of corner matches by the matching method. The maximum corner correspondence should be the average repeatability of the ARCSS detector [10]. Since the AL-based method finds almost the accurate transformation parameters, its corner correspondence should be the maximum. However, it was not found so for transformations comprising rotations followed by cropping.

We had the average results over abovementioned 23 original images and their 7590 transformed images. Fig. 2 shows the corner correspondence by AL-based and DT-based methods along with the detector average repeatability under different geometric transformations and signal processing attacks. The AL-based method performed almost as the average repeatability of the ARCSS detector except for those transformations comprising rotations where some corners had been cropped off. It offered significantly higher corner correspondence than the DT-based method. These results are detailed in Figs. 3(a)-(d).

The significant performance difference in term of corner correspondence between AL-based and DT-based methods is 


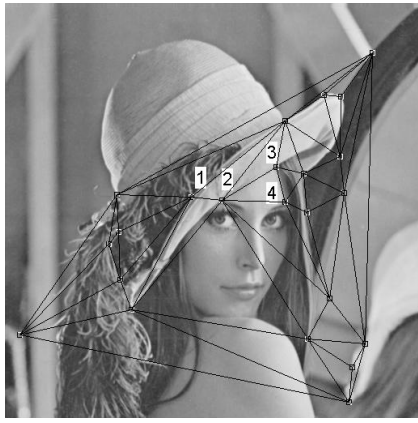

(a) original Lena image

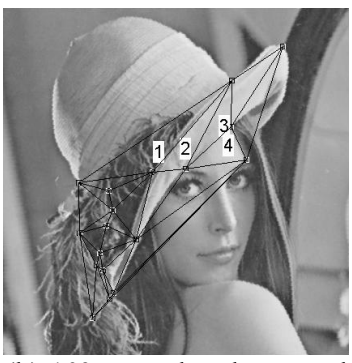

(b) $10^{\circ}$ rotated and cropped
Fig. 4. Corner matching by the existing DT-based method [2].

because of three main reasons. First, the Delaunay triangulation is very much sensitive to outliers. It is unique for a given set of corners but the corner detector often offers a different set of corners under geometric transformations as well as in noising and lossy compression. Therefore, the sets of triangles between original and test images differ considerably. Second, the interior angles used for similarity measurement of Delaunay triangles change according to the strength of the affine transformations, especially when the image is non-uniformly scaled, as shown in Fig. 2 (NU scale and rot.-scale). Finally, the AL-based method finds the affine transformation parameters almost accurately and transforms the original corner set prior to matching with the test corner set. Therefore, it offers corner correspondence almost as the same as the average repeatability of the involved corner detector.

For examples, Fig. 4 shows the corner matching by the DTbased method. Total 18 corners were detected in both the original and $10^{\circ}$ rotated Lena images and the number of repeated corners by the ARCSS detector was 10 . Total 4 triangles and their 6 vertices (corners) were matched by the DT-based method. The matched triangles are shown in Figs. 4(a)-(b) using numbers 1-4. In contrast, Fig. 5 shows the corner matching by the AL-based method using the same pair of images shown in Figs. 4(a)-(b). The estimated angle was $10.29^{\circ}$ and total 10 corners were matched by the proposed method. The matched corners are shown in Figs. 5(a)-(b) using numbers 1-10. Table I presents estimated parameters by the proposed method under some affine transformations on Lena image. It is evident from this table that the AL-based method estimates parameters very precisely and the best matches were found within first five iterations of the outer loop (steps $2-9$ of the matching procedure in Section 3.2).

Note that we also estimated localization [4] of matching corners. To find localization we transformed original corners using original parameters for the DT-based method and using estimated parameters for the AL-based method. Both methods had almost the same localization since they were executed with the same detector.

Table I. Estimated transformation parameters by the proposed AL-based method under some affine transformations on Lena.

\begin{tabular}{c|c|c|c|c|c|c|c}
\hline \hline attack & size & $\theta\left(^{\circ}\right)$ & $s_{x}$ & $s_{y}$ & $t_{x}$ & $t_{y}$ & iteration \\
\hline $\mathrm{R}^{1}$ & $442 \times 442$ & 10.29 & 1.00 & 1.01 & 0.41 & 0.42 & 3 \\
\hline $\mathrm{US}^{2}$ & $359 \times 359$ & 00.36 & 0.71 & 0.71 & -0.31 & -0.09 & 4 \\
\hline $\mathrm{NUS}^{3}$ & $461 \times 564$ & -0.10 & 0.89 & 1.10 & -0.01 & 0.78 & 2 \\
\hline $\mathrm{RS}^{4}$ & $398 \times 398$ & 09.87 & 0.88 & 0.88 & 1.02 & -0.82 & 5 \\
\hline $\mathrm{RST}^{5}$ & $388 \times 393$ & 09.87 & 0.88 & 0.88 & 3.52 & -5.82 & 5 \\
\hline \hline
\end{tabular}

${ }^{1}$ rotation $\theta=10^{\circ} ;{ }^{2}$ uniform scale $s_{x}=s_{y}=0.7 ;{ }^{3}$ non-uniform scale $s_{x}=0.9, \quad s_{y}=1.1 ;{ }^{4}$ rotation-scale $\theta=10^{\circ}, s_{x}=0.9, s_{y}=0.9$; ${ }^{5}$ rotation-scale-trans. $\theta=10^{\circ}, s_{x}=0.9, s_{y}=0.9, t_{x}=10, t_{y}=-5$.

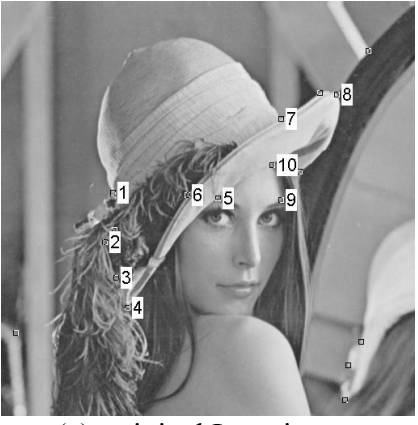

(a) original Lena image



(b) $10^{\circ}$ rotated and cropped
Fig. 5. Corner matching by the proposed AL-based method.

\section{CONCLUSIONS}

The proposed AL-based matching method takes the advantage of the affine-length invariance between corners on the same curve. It also uses the absolute curvature values many of which either remain unaltered or change slightly under affine transformations. We have compared the proposed method with a promising corner matching method based on the Delaunay triangulations considering a wide range of affine transformations. In experiments, the AL-based method offered significantly higher corner correspondence than the DT-based method. Unlike many matching methods in the literature, the AL-based method also offers estimated affine transformation parameters. Future works include investigating its performance in applications such as image retrieval and stereo matching.

\section{REFERENCES}

[1] I. -K. Jung and S. Lacroix, "A robust interest points matching algorithm," in Proc. Int. Conf. on Comp. Vis., 2, 538-543, 2001.

[2] D. Zhou, G. Li, and Y. -H. Liu, "Effective corner matching based on Delaunay triangulation," in Proc. Int. Conf. on Robotics and Automation, 3, 2730-2733, Apr.-May 2004.

[3] E. R. Davies, Machine vision: theory, algorithms, practicalities, Academic Press, London, 1990.

[4] K. Mikolajczyk and C. Schmid, "Scale \& affine invariant interest point detectors," J of Comp. Vis., 60(1), 63-86, Oct. 2004.

[5] R. Horaud and T. Skordas, "Stereo correspondence through feature grouping and maximal cliques," IEEE Trans. on Pat. Anal. and Mach. Intel., 11(11), 1168-1180, Nov. 1989.

[6] W. S. Rutkowski, "Recognition of occluded shapes using relaxation," Comp. Graph. and Img. Process., 19(2), 111-128, Jun. 1982.

[7] N. M. Nasrabadi and W. Li, "Object recognition by a Hopfield neural network," IEEE Trans. on Sys., Man and Cyb., 21(6), 15231535, Nov.-Dec. 1991.

[8] K. -J. Lee, Y. -T. Kim, H. -C. Myung, J. -M. Kim, and Z. Bien, "A corner matching algorithm with uncertainty handling capability," in Int. Conf. on Fuzzy Sys., 3, 1469-1474, Jul. 1997.

[9] J. You, E. Pissaloux, and H. A. Cohen, "A hierarchical image matching scheme based on the dynamic detection of interesting points," in Proc. ICASSP, 4, 2467-2470, May 1995.

[10] M. Awrangjeb, G. Lu, and M. Murshed, "An affine resilient curvature scale-space corner detector," accepted in ICASSP 2007.

[11] J. Canny, "A computational approach to edge detection," IEEE Trans. on Pat. Anal. and Mach. Intel., 8(6), 679-698, 1986.

[12] Acclaim images, http://www.acclaimimages.com. 